\title{
No association between the oligodendrocyte-related gene PLP1 and schizophrenia in the Japanese population
}

\author{
Branko Aleksic $\cdot$ Masashi Ikeda $\cdot$ Ryoko Ishihara $\cdot$ \\ Shinichi Saito · Toshiya Inada · Nakao Iwata • \\ Norio Ozaki
}

Received: 3 April 2008/Accepted: 21 June 2008/Published online: 5 July 2008

(C) The Japan Society of Human Genetics and Springer 2008

\begin{abstract}
P L P 1$ is one of the major myelin-related genes. A large body of expression-based studies showed significantly lower levels of the PLP1 messenger ribonucleic acid (mRNA) transcripts in schizophrenia. Moreover, one family-based study identified a weak association signal in a male subset using 487 Chinese family trios. We carried out a population-based association study between $P L P 1$ and schizophrenia in 1,640 subjects. Our data does not support genetic variation in close vicinity or within PLP1 locus as a susceptibility factor.
\end{abstract}

Keywords Genetic association - Myelination ·

$\mathrm{X}$ chromosome $\cdot$ Proteolipid protein 1

\section{Introduction}

Schizophrenia is a severe mental disorder with a global prevalence of $1 \%$. It is known that a genetic component

B. Aleksic $(\bowtie) \cdot$ R. Ishihara · S. Saito · N. Ozaki

Department of Psychiatry, Graduate School of Medicine,

Nagoya University, Showa-ku, Tsurumai-Cho, 65, Nagoya,

Aichi 466-8550, Japan

e-mail: branko@med.nagoya-u.ac.jp

B. Aleksic $\cdot$ M. Ikeda $\cdot$ N. Iwata

Department of Psychiatry, Graduate School of Medicine,

Fujita Health University, Aichi, Japan

B. Aleksic $\cdot$ M. Ikeda $\cdot$ R. Ishihara $\cdot$ S. Saito $\cdot$

N. Iwata $\cdot$ N. Ozaki

CREST, Japan Science and Technology Agency,

Saitama 332-0012, Japan

T. Inada

Seiwa Hospital, Institute of Neuropsychiatry, Tokyo, Japan is in some degree responsible for its pathogenesis; however, specific factors have not yet been discovered. One hypothesis is that oligodendrocyte dysfunction may confer susceptibility for schizophrenia (Stewart and Davis 2004). This viewpoint is along the lines of a developmental model of etheogenesis, as environmental factors might interfere with cellular maturation of the central nervous system (CNS) (i.e., myelination). Moreover, certain alleles may exhibit protective (or inclining) properties toward impact of the deleterious environmental effect.

PLP1 (length $15.8 \mathrm{kbp}$, location $\mathrm{Xq} 22$ ) is one of the oligodendrocyte-related genes. Proteins encoded by the aforementioned gene (proteolipid protein 1 and its splicing variant DM20) are integral to membranes and together constitute almost $50 \%$ of the protein in CNS myelin. The primary role is thought to be related to the adhesion and stabilization of the extracellular myelin membrane surfaces (Klugmann et al. 1997). Mutations associated with PLPI are responsible for: (1) Pelizaeus-Merzbacher disease (OMIM \#312080) characterized clinically by spastic quadriplegia, ataxia, and developmental delay (Johnson et al. 1991), and (2) spastic paraplegia type 2 (OMIM \#312920).

A large number of expression- and animal-model-based studies have shown significantly lower levels of PLPI messenger ribonucleic acid (mRNA) in schizophrenia (Tkachev et al. 2003; Aston et al. 2004; Le-Niculescu et al. 2007; Sokolov 2007). One study examined the hypothesis of PLP1 as candidate gene (Qin et al. 2005), and data provided evidence for genetic association with schizophrenia. As PLPI is indeed an attractive candidate gene for schizophrenia, we performed a gene-centric (Neale and Sham 2004) association study using a large Japanese sample to replicate previous findings. 
Table 1 Demographic data

\begin{tabular}{llll}
\hline Status & Gender & Number & Average age \\
\hline Case & Male & 418 & $40.27 \pm 11.60$ \\
& Female & 410 & $44.05 \pm 15.10$ \\
Control & Male & 410 & $40.78 \pm 13.76$ \\
& Female & 402 & $43.09 \pm 13.91$ \\
\hline
\end{tabular}

\section{Sample}

Our sample consisted of 828 patients with schizophrenia (average age $42.14 \pm 13.57$ years) and 812 subjects with no personal or family history of psychiatric illness (average age $41.92 \pm 13.87$ years). The subjects consisted of Japanese individuals. Other relevant demographic data are shown in Table 1.

All schizophrenic patients met Diagnostic and Statistical Manual of Mental Disorders, Fourth Edition (DSM IV) criteria, reflected by consensual diagnosis of two experienced psychiatrists. Prior to inclusion in the control set, subjects were screened on the basis of a brief diagnostic interview. Subsequent to the study description, written informed consent was requested from each subject. This study was approved by the ethics committee at Nagoya University.

\section{Methods}

All association analyses were carried out by calculating the $P$ values for each single nucleotide polymorphism (SNP) marker, and significance was determined at the 5\% level using either the chi-square test or Fisher's exact test. All $P$ values are two-sided. Gender was taken into account, with the male subjects contributing a single allele to the analysis and female subjects contributing two alleles. In other words, for an organism where the male is of heterogametic and the female is of homogametic gender, regarding the X-linked genes, males normally possess only one $\mathrm{X}$ chromosome. They are hemizygous for (nearly) all genes that are located on the $\mathrm{X}$ chromosome (such as PLPI). Therefore, the case cohort was stratified by gender and tested against corresponding gender-specific control groups. For the female cohort, association between genotypes and alleles of SNPs and schizophrenia susceptibility were measured. For the male analysis, only allele-wise association was measured.

Linkage disequilibrium (LD) block structure was assessed in accordance with the confidence intervals criteria (Gabriel et al. 2002). Log likelihood ratio tests for assessing haplotype-wise association between schizophrenia and combination of tagging SNPs was performed on the software UNPHASED v3.04 (Dudbridge 2003) with a permutation test for calculating empirical significance levels for differences in haplotype frequencies between case and control sets. Male samples (containing only one PLPI allele) allowed unambiguous assignments of haplotypes. For female subjects, haplotypes and their frequencies were assigned by an estimation maximization algorithm as implemented in UNPHASED v3.04. Due to inherent uncertainty of haplotype prediction for female subjects, these analyses were performed when the case cohort was stratified by gender and tested against corresponding gender-specific control groups.

Power was calculated in accordance with methods described by Skol et al. (2006). In brief, for predefined alpha level and model of inheritance, statistical power of any given sample is a function of sample and effect size. In other words, power is directly proportional to sample size on the one hand and minor allele frequency (MAF) and genotype relative risk (GRR) on the other hand.

To test for genetic association, the gene-based approach was implemented. This method implies inclusion of both gene and gene-adjacent regions in the association study (Neale and Sham 2004). Therefore, the screened region was extended $5 \mathrm{~kb}$ upstream of the annotated transcription start site and downstream at the end of the last $P L P 1$ exon. Later, by taking advantage of observed LD patterns in a predefined region, the most informative subset of SNPs was chosen from an open-access source. Specifically, polymorphisms were selected from the HapMap database (release \#21; phase II; July 2006, population: Japanese in Tokyo) based on correlation coefficient $\left(r^{2}\right)$ between two loci. In other words, if $r^{2}$ was 0.8 or greater, then only one of the two loci was selected for the association study. Moreover, SNPs with a minor allele population frequency less than $5 \%$ were excluded. Based on the aforementioned criteria, three SNPs (rs471416, rs2233696, and rs10521502) were selected for genotyping. The SNP from the initial report (Qin et al. 2005) that had been shown to be associated with schizophrenia (rs475827) failed to met tagging SNP criteria due to low minor allele frequency in the Japanese population (4.7\%). However this SNP was included as well, as it is the SNP that was shown to be associated with schizophrenia. Moreover, analysis of HapMap data showed that allele frequencies of rs475827 in the Chinese population $(\mathrm{MAF}=4.4 \%)$ were similar to those in the Japanese population $(\mathrm{MAF}=4.7 \%)$. Analysis of LD pattern and distribution of common haplotypes for the SNPs $(\mathrm{MAF}>5 \%$ ) in the PLPI region (HapMap data) provided comparable results for both populations.

For SNPs on the X chromosome, assessment of deviation from Hardy-Weinberg equilibrium (HWE) was different. In detail, HWE can be tested using two null hypotheses: (1) allele frequencies between cases and controls are equal, and (2) HWE holds in females (Zheng et al. 2007). 
Genomic deoxyribonucleic acid (DNA) was extracted from peripheral blood. Allelic discrimination assay (ABI Biosystem) was carried out for genotype characterization of the selected SNPs. For each 384-well plate, three nontemplate controls were included.

\section{Results}

Regarding tagging SNPs, a priori power analysis showed that our sample reached a level of $80 \%$ for detecting association under the multiplicative model of inheritance when GRR was set to 1.35 for gender-stratified data and MAF was $30 \%$ (which is the lowest MAF for tagging SNPs). In case of the SNP from the initial study that was shown to be associated with schizophrenia, our sample had $80 \%$ power for detecting association when GRR was set to 1.8 for gender-stratified data and MAF was set to $4 \%$.

A priori evaluation of HapMap data regarding the PLP1related region showed existence of one LD block spreading gene region. The same pattern was observed a posteriori by assessing our experimental data. Analyses were carried out using Haploview v3.32 (Barrett et al. 2005). Deviation from HWE was not detected. Statistical analysis did not provide sufficient evidence for the genetic association (Tables 2, 3, 4).

\section{Discussion}

The common disease-common variant hypothesis states that multigenetic diseases, such as schizophrenia, that are evolutionarily neutral (i.e., a little or no effect on reproductive fitness) during human history may be significantly influenced by common variants (Lander 1996). Therefore, if an allelic variant at the disease susceptibility locus is responsible for predisposition to a common complex disease, then allele-, genotype-, or haplotype-wise association tests will detect such variants (or a tagging SNP that is in LD with a deleterious allele).

The first and only indication that the $P L P 1$-related region harbors variation that might influence susceptibility to schizophrenia was provided by Qin and colleagues, who identified a weak association signal between rs475827 (intronic SNP, MAF $=4.4 \%$ ) and schizophrenia in a male cohort only using 487 Chinese family trios. However, our study, based on adequate statistical power, did not support association between schizophrenia and the PLPI locus. As lack of replication may also be falsely negative, possibly due to the lack of adequately powered sample sizes, we performed a power analysis of the sample from the initial

Table 3 Results ( $P$ values single marker)

\begin{tabular}{llll}
\hline dbSNP & Males & Females & \\
\cline { 3 - 4 } & Allele & Allele & Genotype \\
\hline rs475827 & 0.439 & 0.205 & 0.314 \\
rs471416 & 0.943 & 0.959 & 0.573 \\
rs2233696 & 0.276 & 0.381 & 0.626 \\
rs10521502 & 0.596 & 0.559 & 0.634 \\
\hline
\end{tabular}

Table 4 Results ( $P$ value multimarker analysis, sliding window)

\begin{tabular}{|c|c|c|c|c|c|c|}
\hline \multirow[t]{2}{*}{ dbSNP } & \multicolumn{3}{|l|}{ Males } & \multicolumn{3}{|l|}{ Females } \\
\hline & 2 SNPs & 3 SNPs & 4 SNPs & 2 SNPs & 3 SNPs & 4 SNPs \\
\hline rs475827 & 0.685 & 0.682 & 0.881 & 0.929 & 0.817 & 0.796 \\
\hline rs 471416 & 0.585 & & & 0.877 & & \\
\hline rs 2233696 & & 0.844 & & & 0.751 & \\
\hline rs10521502 & 0.747 & & & 0.826 & & \\
\hline
\end{tabular}

SNP single nucleotide polymorphism

Table 2 Genotype distribution

\begin{tabular}{|c|c|c|c|c|c|c|c|c|c|c|}
\hline \multirow[t]{3}{*}{ dbSNP } & \multirow[t]{3}{*}{ Status } & \multirow[t]{3}{*}{ Allele $(p>q)$} & \multirow[t]{3}{*}{ Position } & \multirow{2}{*}{\multicolumn{2}{|c|}{$\begin{array}{l}\text { Males } \\
\text { Allele }\end{array}$}} & \multicolumn{5}{|c|}{ Females } \\
\hline & & & & & & \multicolumn{3}{|c|}{ Genotype } & \multicolumn{2}{|c|}{ Allele } \\
\hline & & & & $p /-$ & $q /-$ & $p / p$ & $p / q$ & $q / q$ & $p$ & $q$ \\
\hline \multirow[t]{2}{*}{ rs475827 } & Case & $A>G$ & Upstream & 25 & 393 & 2 & 60 & 348 & 64 & 756 \\
\hline & Control & & & 19 & 391 & 2 & 45 & 355 & 49 & 755 \\
\hline \multirow[t]{2}{*}{ rs471416 } & Case & $\mathrm{C}>\mathrm{T}$ & Upstream & 254 & 164 & 158 & 181 & 71 & 497 & 323 \\
\hline & Control & & & 251 & 159 & 147 & 192 & 63 & 486 & 318 \\
\hline \multirow[t]{2}{*}{ rs2233696 } & Case & $A>G$ & Intronic & 141 & 277 & 55 & 183 & 172 & 293 & 527 \\
\hline & Control & & & 154 & 256 & 58 & 189 & 155 & 305 & 499 \\
\hline \multirow[t]{2}{*}{ rs10521502 } & Case & $\mathrm{C}>\mathrm{T}$ & Downstream & 130 & 288 & 46 & 167 & 197 & 259 & 561 \\
\hline & Control & & & 120 & 290 & 44 & 177 & 181 & 265 & 539 \\
\hline
\end{tabular}


report (Qin et al. 2005). This analysis showed that the sample used in our research had a higher level of power. In other words, regarding our sample, to detect association when minor allele frequency was set to population-based data (rs475827), in order to achieve $80 \%$ of power, GRR has to be more than 1.8 (for gender-stratified data). On the other hand, regarding the schizophrenia-associated SNP rs475827, sample $\left(N_{\text {male trios }}=253\right)$ in the original report (Qin et al. 2005), the same minor allele frequency and GRR had only $51 \%$ of power for detecting association.

In light of the fact that a large body of expression-based studies have shown significantly lower levels of PLPI mRNA transcripts in animal models of schizophrenia or in brain tissue from deceased schizophrenic patients in comparison with healthy controls (Tkachev et al. 2003; Aston et al. 2004; Le-Niculescu et al. 2007), our results suggest that the downregulation is not influenced by the predisposing common genetic variants within or in close vicinity to the PLPl gene region.

An emerging body of work over the last 5 years has implicated myelin/glia related dysfunction in schizophrenia (Tkachev et al. 2003; Aston et al. 2004; Le-Niculescu et al. 2007). Moreover, downregulation of at least some of the many abnormally expressed myelin-associated genes in schizophrenia might be influenced by a systematic mechanism such as methylation, as in case of SOX10 (Iwamoto et al. 2005), or a transcriptional factor that can exert an effect on multiple myelin-associated genes such as $Q K I$ (Aberg et al. 2006; Haroutunian et al. 2006) rather than an SNP in the regulatory region of each abnormally expressed myelin-associated genes. Interestingly, Aberg and colleagues showed that human $Q K I$ mRNA levels can account for a high proportion of normal interindividual mRNA expression variation (and covariation) of PLPI. Therefore, it is not surprising that we could not replicate the finding from the original report.

The chances are that new experimental methodologies (such as oligonucleotide arrays) will eventually increase signal-to-noise ratio and give us a better perspective regarding genetic factors influencing complex disorders and probably higher reproducibility of the results from genetic association studies.

Acknowledgments This work was supported in part by research grants from the Ministry of Education, Culture, Sports, Science and Technology of Japan, the Ministry of Health of Japan, Labor and Welfare, Grant-in-Aid for Scientific Research on Pathomechanisms of Brain Disorders from the Ministry of Education, Culture, Sports, Science and Technology of Japan, MEXT Academic Frontier, the Japan Health Sciences Foundation (Research on Health Sciences focusing on Drug Innovation), and the Core Research for Evolutional Science and Technology.

Conflict of interest statement None declared.

\section{References}

Aberg K, Saetre P, Jareborg N, Jazin E (2006) Human QKI, a potential regulator of mRNA expression of human oligodendrocyte-related genes involved in schizophrenia. Proc Natl Acad Sci USA 103:7482-7487

Aston C, Jiang L, Sokolov BP (2004) Microarray analysis of postmortem temporal cortex from patients with schizophrenia. J Neurosci Res 77:858-866

Barrett JC, Fry B, Maller J, Daly MJ (2005) Haploview: analysis and visualization of LD and haplotype maps. Bioinformatics 21:263265

Dudbridge F (2003) Pedigree disequilibrium tests for multilocus haplotypes. Genet Epidemiol 25:115-121

Gabriel SB, Schaffner SF, Nguyen H, Moore JM, Roy J, Blumenstiel B, Higgins J, DeFelice M, Lochner A, Faggart M, Liu-Cordero SN, Rotimi C, Adeyemo A, Cooper R, Ward R, Lander ES, Daly MJ, Altshuler D (2002) The structure of haplotype blocks in the human genome. Science 296:2225-2229

Haroutunian V, Katsel P, Dracheva S, Davis KL (2006) The human homolog of the QKI gene affected in the severe dysmyelination "quaking" mouse phenotype: downregulated in multiple brain regions in schizophrenia. Am J Psychiatry 163:1834-1837

Iwamoto K, Bundo M, Yamada K, Takao H, Iwayama-Shigeno Y, Yoshikawa T, Kato T (2005) DNA methylation status of SOX10 correlates with its downregulation and oligodendrocyte dysfunction in schizophrenia. J Neurosci 25:5376-5381

Johnson VP, Carpenter NJ, Kelts KA (1991) Pelizaeus-Merzbacher disease: clinical and DNA-linkage study of an extended family. Am J Med Genet 41:355-361

Klugmann M, Schwab MH, Puhlhofer A, Schneider A, Zimmermann F, Griffiths IR, Nave KA (1997) Assembly of CNS myelin in the absence of proteolipid protein. Neuron 18:59-70

Lander ES (1996) The new genomics: global views of biology. Science 274:536-539

Le-Niculescu H, Balaraman Y, Patel S, Tan J, Sidhu K, Jerome RE, Edenberg HJ, Kuczenski R, Geyer MA, Nurnberger JI Jr, Faraone SV, Tsuang MT, Niculescu AB (2007) Towards understanding the schizophrenia code: an expanded convergent functional genomics approach. Am J Med Genet B Neuropsychiatr Genet 144:129-158

Neale BM, Sham PC (2004) The future of association studies: genebased analysis and replication. Am J Hum Genet 75:353-362

Qin W, Gao J, Xing Q, Yang J, Qian X, Li X, Guo Z, Chen H, Wang L, Huang X, Gu N, Feng G, He L (2005) A family-based association study of PLP1 and schizophrenia. Neurosci Lett 375:207-210

Skol AD, Scott LJ, Abecasis GR, Boehnke M (2006) Joint analysis is more efficient than replication-based analysis for two-stage genome-wide association studies. Nat Genet 38:209-213

Sokolov BP (2007) Oligodendroglial abnormalities in schizophrenia, mood disorders and substance abuse. Comorbidity, shared traits, or molecular phenocopies? Int J Neuropsychopharmacol 10:547555

Stewart DG, Davis KL (2004) Possible contributions of myelin and oligodendrocyte dysfunction to schizophrenia. Int Rev Neurobiol 59:381-424

Tkachev D, Mimmack ML, Ryan MM, Wayland M, Freeman T, Jones PB, Starkey M, Webster MJ, Yolken RH, Bahn S (2003) Oligodendrocyte dysfunction in schizophrenia and bipolar disorder. Lancet 362:798-805

Zheng G, Joo J, Zhang C, Geller NL (2007) Testing association for markers on the X chromosome. Genet Epidemiol 31:834-843 